\title{
Universal active metasurfaces for dynamic beam steering and reconfigurable focusing at telecommunication wavelengths
}

\author{
Ghazaleh Kafaie Shirmanesh ${ }^{1}$, Ruzan Sokhoyan ${ }^{1}$, Pin Chieh $W_{u^{1,2}}$ and Harry A. Atwater ${ }^{1,3}$ \\ ${ }^{1}$ Thomas J. Watson Laboratory of Applied Physics, California Institute of Technology, Pasadena, CA 91125, USA. \\ ${ }^{2}$ Department of Photonics, National Cheng Kung University, Tainan 70101, Taiwan. \\ ${ }^{3}$ Kavli Nanoscience Institute, California Institute of Technology, Pasadena, CA 91125, USA. \\ Author e-mail address: ghazaleh@caltech.edu
}

\begin{abstract}
We report the design and experimental demonstration of a 'universal' reconfigurable metasurface, which by electrical control of individual metasurface elements, enables both dynamic beam steering and reconfigurable focusing. $\odot 2020$ The Author(s)
\end{abstract}

Recent advances in nanophotonic integration have created burgeoning interest in development of metasurfaces to demonstrate compact, high-performance, and low-cost optical devices. Metasurfaces enable control of optical amplitude, phase and polarization, leading to precise engineering of the wave front [1]. Until now, metasurfaces have mostly been reported in bespoke designs, tailored to particular applications where optical function is encoded at the time of structure fabrication. Tunable metasurfaces have recently been explored as a route to dynamical post-fabrication control of the constitutive properties of scattered light, where phase and amplitude are modulated via electrical bias, optical pumping, heating, or elastic strain [2]. However to date, active metasurfaces have been designed to achieve a single function, and have lacked a sufficiently powerful device and array architecture to realize general design paradigm for universal tunability where any desired scattering wave front can be achieved, using a single programmable hardware component, in analogy with field-programmable gate arrays in electronics.

Here, we report a prototypical universal metasurface that can provide multiple functions using a single hardware device. In our universally reprogrammable metasurface, the phase response of the individual metasurface elements can be electronically controlled via application of an external bias voltage. By integrating a transparent conducting oxide (TCO) layer into the metasurface, field-effect-induced modulation of the refractive index of the metasurface elements could be achieved when applying bias. Such electrical tuning has been proven to be a robust, energy-efficient and reversible scheme for tuning active metasurfaces [3, 4]. By precisely tailoring the phase profile of the electro-optically tunable metasurface, we realize a device that can exhibit multiple functions in the near infrared (NIR) wavelength range.

Figure 1(a) shows a schematic of the proposed metasurface. The schematic of the metasurface unit cell is depicted in Fig. 1(b). The metasurface consists of a gold back reflector, followed by a dielectric spacer layer. On top of the dielectric spacer layer, we deposit an actively tunable indium-tin-oxide (ITO) layer, followed by a gate dielectric, and gold nanoantennas. When a direct current (DC) electric bias is applied between the ITO layer and the nanoantennas, the ITO layer undergoes a reversible field-effect-induced index change. In other words, the ITO charge carrier density close to the interface of the ITO and the gate dielectric can be tuned by changing the applied electric field. When the applied bias is further increased, an epsilon-near-zero (ENZ) condition holds in an accumulation layer located within ITO. When spectrally overlapping the magnetic dipole resonance of the metasurface with the ENZ regime of the ITO layer, the metasurface exhibits large phase modulation (Fig. 1(c)). As seen in Fig. 1(c), when the external bias is changed, we observe a reflectance change that is accompanied by a significant phase modulation of around $270^{\circ}$.

(a)

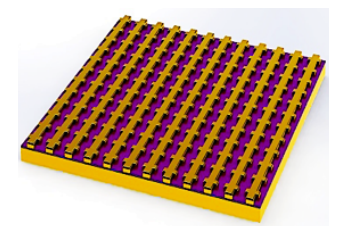

(b)

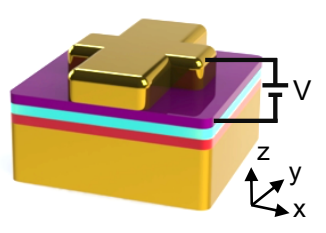

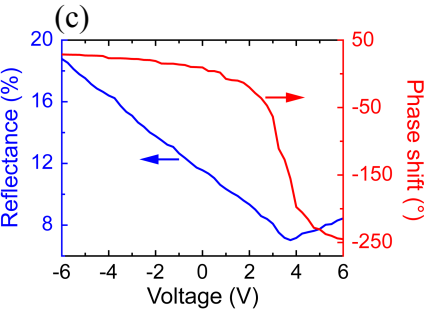

Fig. 1. (a) Schematic of the proposed metasurface. (b) Schematic of the unit cell of the metasurface. (c) Measured reflectance (blue curve) and phase shift (red curve) as a function of applied bias voltage.

Controlling the bias voltages applied to independently-addressable metasurface elements would enable dynamic control of the wave front of the reflected light via a pixel-by-pixel reconfiguration. The ability to actively 
control the wave front of the reflected light implies that the functionality of the metasurface can be reprogrammed. In the present work, we experimentally demonstrate that depending on the bias application configuration, our universal metasurface can act as both a dynamic beam steering device and a tunable cylindrical metalens with reconfigurable focal length and numerical aperture. The fabricated universal metasurface consists of 96 individually addressable metasurface elements. We realized beam steering by using a blazed grating approach. Figures 2(a) and 2(b) show the spatial profiles of the phase of the light reflected from the metasurface, which correspond to steering angles of $22.2^{\circ}$ and $7.4^{\circ}$. By discretizing the phase shift introduced by the metasurface pixels into four levels $0^{\circ}, 90^{\circ}, 180^{\circ}$, and $270^{\circ}$, and then changing the pixel repetition number $(\mathrm{RN})$ for each phase shift value within one supercell, we electrically modulated the effective periodicity of the metasurface array. By employing the spatial phase profile illustrated in Fig. 2(a) (Fig. 2(b)), we measured the far-field radiation pattern shown in Fig. 2(c) (Fig. 2(d)). As seen in Figs. 2(c) and 2(d), electronically altering the effective period of the metasurface resulted in a shift of the spatial position of the first diffraction order, enabling manipulation of the far-field radiation pattern.

(a)

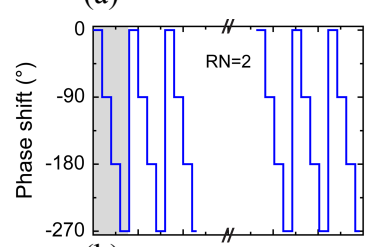

(b)

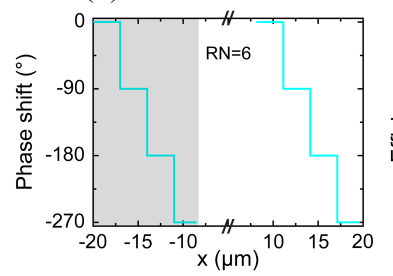

(c)

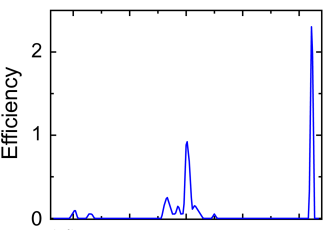

(d)

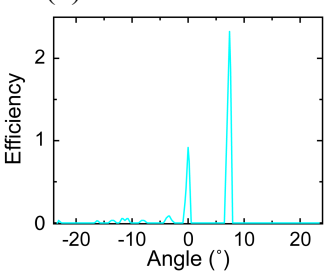

(e)
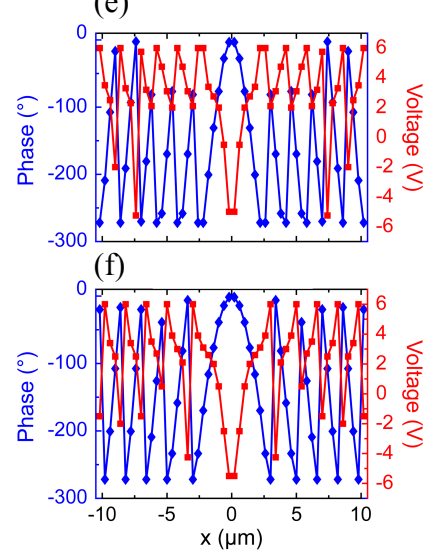

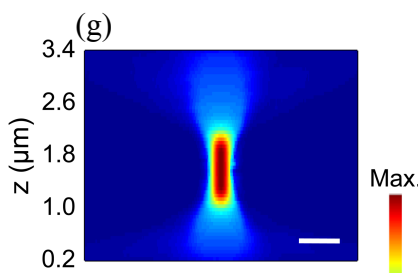

(h)

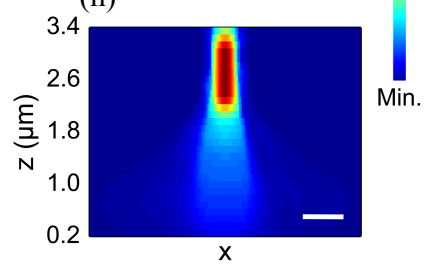

Fig. 2. (a) and (b) Spatial phase distributions of the metasurface elements with $R N$ values of (a) $R N=2$ and (b) $R N=6$ used for creating blazed grating phase gradients. (c) and (d) Experimentally measured far field radiation pattern as a function of the steering angle for RN values of (c) $\mathrm{RN}=2$ and (d) $\mathrm{RN}=6$. (e) and (f) Spatial phase (diamond) and voltage (square) distribution of a metalens with focal lengths of (e) $f$ $=1.5 \mu \mathrm{m}$ and (f) $f=3 \mu \mathrm{m}$. (g) and (h) Measured intensity profile of the beam reflected from the metalens with focal lengths of (g) $f=1.5 \mu \mathrm{m}$ and (h) $f=3 \mu \mathrm{m}$. Scale bar is $2 \mu \mathrm{m}$.

Using the same concept of controlling the phase imposed by each individual metasurface pixel, we were able to demonstrate that our universal metasurface could act as a tunable cylindrical metalens. For the experimental demonstration, we developed the spatial distribution of the phase shift (diamond) and the corresponding applied bias voltage (square) required to focus the reflected beam at focal lengths of $1.5 \mu \mathrm{m}$ (Fig. 2(e)) and $3 \mu \mathrm{m}$ (Fig. 2(f)). We then experimentally characterized the dynamic focusing performance of the metasurface by extracting the cross sections of the captured $x y$-plane intensity profiles at different $z$ positions. Figures $2(\mathrm{~g})$ and $2(\mathrm{~h})$ illustrate the reflected beam intensity profiles in the $x z$-plane for the bias application configurations shown in Figs. 2(e) and 2(f), respectively. As seen in Figs. 2(g) and 2(h), the proposed metasurface can focus the reflected beam at the desired reconfigurable depths.

In conclusion, we proposed an electrically-tunable universal metasurface in the NIR wavelength range. The observed tunable optical response is based on the field-effect-induced modulation of the complex refractive index of the TCO layer incorporated into the metasurface. By designing the phase response of the independently-addressable metasurface pixels, we used the same device to demonstrate beam steering and dynamic focusing.

\section{References}

1. H.H. Hsiao, C.H. Chu, and D.P. Tsai, "Fundamentals and applications of metasurfaces," Small Methods 1, 1600064 (2017).

2. A.M. Shaltout, V.M. Shalaev, and M.L. Brongersma, "Spatiotemporal light control with active metasurfaces," Science 364, eaat3100 (2019).

3. Y.-W. Huang, et al., "Gate-tunable conducting oxide metasurfaces,” Nano Lett. 16, 5319-5325 (2016).

4. G.K. Shirmanesh, R. Sokhoyan, R.A. Pala, and H.A. Atwater, "Dual-gated active metasurface at $1550 \mathrm{~nm}$ with wide $\left(>300^{\circ}\right)$ phase tunability," Nano Lett. 18, 2957-2963 (2018). 